\title{
Effects of redundant visual stimuli on temporal order judgments
}

\author{
JEFF MILLER \\ University of Otago, Dunedin, New Zealand \\ and \\ EVA KÜHLWEIN and ROLF ULRICH \\ University of Tübingen, Tübingen, Germany
}

\begin{abstract}
Four experiments were conducted in order to compare the effects of stimulus redundancy on temporal order judgments (TOJs) and reaction times (RTs). In Experiments 1 and 2, participants were presented in each trial with a tone and either a single visual stimulus or two redundant visual stimuli. They were asked to judge whether the tone or the visual display was presented first. Judgments of the relative onset times of the visual and the auditory stimuli were virtually unaffected by the presentation of redundant, rather than single, visual stimuli. Experiments 3 and 4 used simple RT tasks with the same stimuli, and responses were much faster to redundant than to single visual stimuli. It appears that the traditional speedup of RT associated with redundant visual stimuli arises after the stimulus detection processes to which TOJs are sensitive.
\end{abstract}

In simple reaction time (RT) tasks (e.g., Donders, 1868/ 1969), participants are required to respond as quickly as possible to the presentation of any stimulus. In these tasks, responses are faster, on average, when two stimuli are presented than when only one is presented (e.g., Raab, 1962; Todd, 1912, cited in Hershenson, 1962). This speedup with redundant stimuli is often called the redundant stimulus effect (RSE), and it is a very general phenomenon. It has been observed with redundant visual stimuli (e.g., Miller, 1982; Schwarz \& Ischebeck, 1994) and with redundant stimuli in two or three different modalities (e.g., Diederich, 1992; Diederich \& Colonius, 1987; Forster, Cavina-Pratesi, Aglioti, \& Berlucchi, 2002; Raab, 1962; Todd, 1912). Analogous speedups with redundant stimuli have also been observed in go/no-go tasks (e.g., Mordkoff \& Yantis, 1991) and in choice RT tasks (e.g., Grice, Canham, \& Boroughs, 1984).

Although the RSE is not very surprising, the reasons for it are still unclear. Research into the causes of the RSE have focused on two main questions, neither of which

This research was supported by a grant from the Royal Society of New Zealand (Marsden U00206) to J.M. and by cooperative research funds from the Deutsche Raum- und Luftfahrtgesellschaft e.V. The authors thank Hans Colonius, J. Toby Mordkoff, James Townsend, and two anonymous reviewers for helpful comments on the manuscript. Correspondence concerning this article should be addressed to J. Miller, Department of Psychology, University of Otago, Dunedin, New Zealand, or to Rolf Ulrich, Abteilung für Allgemeine Psychologie und Methodenlehre, Psychologisches Institut, Universität Tübingen, Friedrichstr. 21, 72072 Tübingen, Germany (e-mail: miller@psy.otago.ac.nz or ulrich@, uni-tuebingen.de).

Note-This article was accepted by the previous editorial team, headed by Neil Macmillan. has yet been answered definitively. First, what is the locus of the RSE - that is, which processes operate more quickly in the presence of redundant stimuli? For example, are perceptual processes more rapid with redundant stimuli than with single stimuli? Alternatively, does the effect instead arise during motor processing (cf. Diederich \& Colonius, 1987; Giray \& Ulrich, 1993; Miller, U1rich, \& Lamarre, 2001; Mordkoff, Miller, \& Roch, 1996)? Second, what is the nature of the facilitation by which two redundant stimuli produce faster responses than does a single stimulus? One possibility is that redundant stimuli simply produce statistical facilitation (e.g., Raab, 1962). Alternatively, the activations produced by the two stimuli could sum (in whichever stage), so that a criterion activation would be reached more rapidly when two stimuli are presented than when only one is (e.g., Miller, 1982; Schwarz, 1989, 1994; see Townsend \& Nozawa, 1995, for an in-depth analysis). Yet other explanations posit crosstalk between channels, so that the presence of one stimulus affects the time needed for detection of the other (e.g., Bernstein, 1970; Mordkoff \& Yantis, 1991).

Raab (1962) was the first to suggest a detailed model for the RSE, and his model included answers to both of these questions. Raab suggested that the RSE is a perceptual effect, caused by faster stimulus detection in trials with redundant stimuli than in trials with single stimuli. Todd (1912) also interpreted his early results as evidence that the RSE arises at the perceptual stage, suggesting that the excitatory effects of different stimuli summate at the perceptual level, and others have since interpreted RSEs in much the same way (e.g., Blake, Martens, Garrett, \& Westendorf, 1980). Moreover, Raab proposed that 
the effect arises because of statistical facilitation. In his model, all stimuli are detected separately, and in redundant trials, the response is initiated as soon as the first one is detected. That is, RT is determined by the latency of a single detection process if only one stimulus is presented, whereas it is determined by the winner of a race between two detection processes in redundant trials. Under very general conditions, the average time for the winner of the race will be shorter than the average time for either racer (see Miller \& Ulrich, 2003).

Although Raab's (1962) model explains the speedup in mean RT caused by redundant stimuli, subsequent research has revealed that the idea of statistical facilitation does not explain observed RSEs at the level of RT distributions. According to race models such as Raab's, observed RT distributions should obey the so-called race model inequality (Miller, 1982; Ulrich \& Giray, 1986), in which

$$
F_{\mathrm{r}}(t) \leq F_{1}(t)+F_{2}(t)
$$

for every value of $t$, where $F_{1}$ and $F_{2}$ are the cumulative probability distributions (CDFs) of RT in the two singlestimulus conditions and $F_{\mathrm{r}}$ is the CDF of RT in the redundant-stimulus condition. Specifically, this inequality holds for all separate-activations race models, in which the processes detecting the two possible stimuli operate separately and each operates at the same speed regardless of whether the other signal is presented (Ashby \& Townsend, 1986; Luce, 1986). Within such models, $F_{\mathrm{r}}(t)$ can approach $F_{1}(t)+F_{2}(t)$ for small values of $t$ if the detection times of the two single signals have a strong negative correlation (Colonius, 1990), but even in that case, Inequality 1 must still hold.

Contrary to race models, observed RT distributions often violate the race model inequality for relatively small values of $t$ (e.g., Diederich, 1992; Egeth \& Mordkoff, 1991; Grice et al., 1984; Miller, 1982; Mordkoff et al., 1996). Apparently, then, processing in redundantstimulus trials is not simply a race between two separate processes of the same sort seen in single-stimulus trials. Instead, it appears that information from both stimuli is somehow combined together to activate the response, resulting in more than mere statistical facilitation. Such a combination can sometimes be explained in terms of cross-channel contingencies and cross-channel facilitation of racers, as in the interactive race model of Mordkoff and Yantis (1991, 1993). In addition, several so-called coactivation models have been developed to describe information combination and the facilitation that results from it, beyond that attributable to cross-channel contingencies (e.g., Miller, 1982; Miller \& Ulrich, 2003; Schwarz, 1989, 1994). Townsend and Nozawa (1995, 1997) presented a more general characterization of both the race model inequality and the models that do and do not satisfy it. So far, however, there have been no decisive attempts to discriminate among the alternative models.

In contrast to his suggestion that the RSE is produced by statistical facilitation, Raab's (1962) proposal that the RSE arises during perceptual processing has proven dif- ficult to test. Some studies in which a perceptual locus of the RSE has been investigated have compared the effects of identical versus different redundant stimuli. If the RSE has a perceptual locus, it would seem reasonable to expect a larger RSE with identical stimuli. Consistent with this expectation, Schwarz and Ischebeck (1994) found evidence of a larger RSE for two parallel lines than for two orthogonal ones. Similarly, Mordkoff and Miller (1993) found larger violations of the race model inequality with two identical target letters than with two different ones, at least after some practice. These data suggest that the RSE is at least partially perceptual, but they are not definitive for two reasons: (1) The speed of perceptual processing was not actually measured, and (2) the observed patterns might arise during a decision process, rather than during a perceptual one, if two identical stimuli activate the detection decision more strongly than do two different ones.

The possibility of a perceptual locus of the RSE has also been examined with psychophysiological methods. For example, in experiments with presentation of visual stimuli to the left or right visual field or to both, eventrelated potentials (ERPs) in redundant trials differed from the sums of ERPs in single-target trials within $100 \mathrm{msec}$ of stimulus onset, consistent with the idea of early perceptual interactions (Miniussi, Girelli, \& Marzi, 1998; Murray, Foxe, Higgins, Javitt, \& Schroeder, 2001). This is even less direct evidence that the RSE results from a perceptual speedup, however, both because it is difficult to relate the size of ERPs to the speed of processing (Meyer, Osman, Irwin, \& Yantis, 1988) and because strong assumptions are needed to justify the comparison of ERPs from the redundant condition against the sum of the ERPs from the two single-stimulus conditions.

As an alternative to the hypothesis of a perceptual locus of the RSE, researchers have, in a number of studies, examined the possibility that the RSE might result instead (or in addition) from a speedup in motor processing. Diederich and Colonius (1987), Giray and Ulrich (1993), and Plat, Praamstra, and Horstink (2000) all found evidence that motor processes operate differently in trials with redundant, as opposed to single, stimuli (e.g., a larger response force is produced), but none found that the motor processes operated more rapidly in these trials. In contrast, Miller et al. (2001) and Mordkoff et al. (1996) used psychophysiological techniques to measure the duration of motor processes directly, and they concluded that these processes had the same duration with redundant stimuli as with single stimuli, contrary to the hypothesis of a motor locus of the RSE. A similar conclusion was reached by Cavina-Pratesi, Bricolo, Prior, and Marzi (2001) on the basis of studies in which the stop signal paradigm was used (cf. Logan, 1994). They found that simple RT responses to redundant signals are less likely to be stopped than responses to single signals and, also, that redundant stop signals are more likely to inhibit some other response than are single stop signals. Both of these findings suggest that redundancy conveys an advantage to premotor processes. 
The question of the locus of the RSE is clearly a difficult one. Redundant stimuli may speed processing at more than one stage of processing, and the affected stages could depend on the task (e.g., stimuli and responses). For example, redundancy gain seems to be greater when redundant stimuli are presented in different sensory modalities than when they are presented in the same modality (e.g., Forster et al., 2002), raising the possibility that between- and within-modality redundancy gain may be produced somewhat differently. Perhaps most seriously, though, attempts to localize the effects of redundant stimuli on the speed of processing in individual stages are hampered by the difficulty of isolating the time consumed by particular stages within the overall RT, which necessarily includes both perceptual and motor components.

The goal of the present experiments was to obtain a relatively direct measure of the influence of redundant stimuli on the speed of certain perceptual processes by using a temporal order judgment (TOJ) task. In TOJ tasks, observers are presented with two stimuli-say, $S_{A}$ and $S_{B}$-in each trial. The time interval from the onset of $S_{A}$ to the onset of $S_{B}$ (i.e., the stimulus onset asynchrony, or SOA) varies randomly from trial to trial, and participants are asked to make an unspeeded judgment of which stimulus appeared first. The responses from a TOJ task are typically used to construct a psychometric function relating response probability to SOA (see, e.g., Figure 1). As the time from the onset of $S_{A}$ to the onset of $S_{B}$ increases, so too does the probability that the observer makes the response that $S_{A}$ appeared first.

TOJ tasks have often been used to test for effects of stimulus properties on the time needed for the detection of a stimulus. For example, Tappe, Niepel, and Neumann (1994) used a constant $2300-\mathrm{Hz}$ tone as $S_{A}$ in all trials. $S_{B}$ was a visual stimulus, and its spatial frequency varied

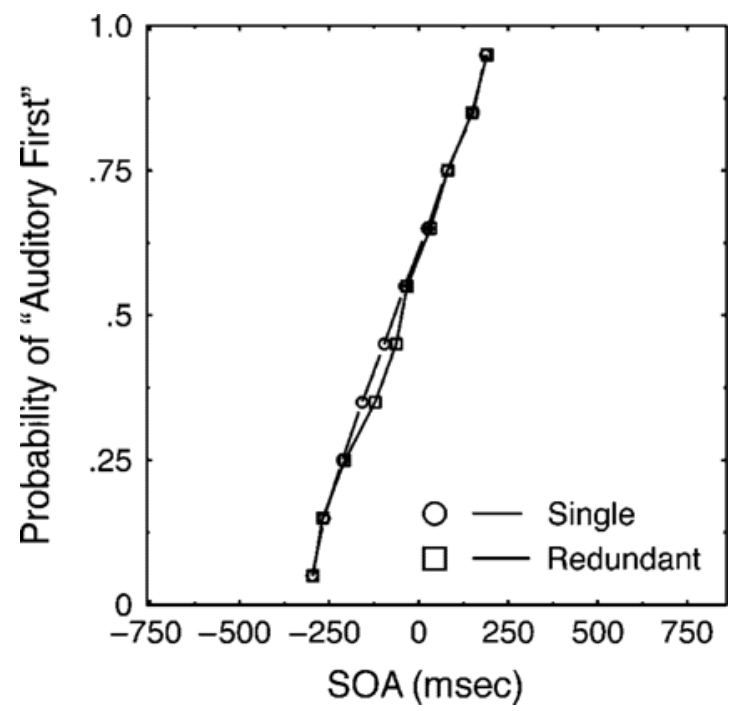

Figure 1. Vincentized average psychometric functions for single and redundant visual stimuli in Experiment 1. across trials. They reasoned that if the latency of perceptual detection depended on spatial frequency, then at any given SOA, the probability of the " $S_{A}$ first" response should depend on the spatial frequency of the visual stimulus. Indeed, this is exactly the result that was obtained in their second experiment, and they interpreted it as evidence that low spatial frequency stimuli are detected more rapidly than high spatial frequency stimuli (cf. Parker \& Dutch, 1987). Note that this conclusion does not require the assumption that only perceptual processes contribute to TOJs; some postperceptual decision process are surely also involved (Sternberg \& Knoll, 1973). Instead, the conclusion simply depends on the assumption that the perceptual latencies used for the TOJ are sensitive to manipulations of the speed with which a stimulus can be detected. In keeping with that assumption, many other perceptual manipulations have also been found to affect TOJs, including stimulus intensity (e.g., Menendez \& Lit, 1983; Roufs, 1963; Sanford, 1971), stimulus duration (Jaśkowski, 1992), stimulus rise time (Jaśkowski, 1993), stimulus motion (Craig \& Busey, 2003), inhibition of return (Gibson \& Egeth, 1994), and directed attention (e.g., Shore, Spence, \& Klein, 2001; Spence, Shore, \& Klein, 2001; Stelmach \& Herdman, 1991).

It is straightforward to use the logic of the TOJ task to check for an effect of redundant stimuli on the time needed for perceptual detection. In each trial of our TOJ task, participants were presented with a constant auditory stimulus and a visual display consisting of a white rectangle to the left of fixation, a white rectangle to the right of fixation, or both rectangles. They were asked simply to indicate in each trial whether the visual display came on before the auditory stimulus, and we tabulated their responses for each type of visual stimulus at each SOA.

The major empirical question addressed by these experiments is whether the single and the redundant visual stimuli yield identical or different psychometric functions for the TOJs. If participants perceive two stimuli more quickly than one, then at a given SOA, they should be more likely to say that the visual display started first when that display has two stimuli than when it has only one. Such an effect would provide strong support for the idea that the RSE has an at least partly perceptual locus. Indeed, such an effect seems quite likely given that (1) adding a second target increases the total energy of the visual display and (2) TOJs are sensitive to stimulus energy.

Alternatively, suppose that the perceptual processes contributing to the TOJ are unaffected by redundancy (i.e., participants perceive one or two stimuli at the same latency, on average). In that case, the probability that the visual display will be perceived as starting before the auditory stimulus should be the same for single and redundant visual stimuli, and therefore, the psychometric functions obtained in these two conditions should be identical. This result would be evidence against the hypothesis of a perceptual locus of the RSE.

Of course, the use of the TOJ task to make inferences about the speed of perceptual processing in a simple RT 
task requires the assumption that an initial common perceptual pathway is used in both tasks, as has been acknowledged by all researchers using this technique (e.g., Sternberg \& Knoll, 1973; Tappe et al., 1994). This assumption seems extremely plausible: Surely, the initial sensory registration of visual stimuli on the retina and a great deal of subsequent visual processing would be independent of the task being performed. Thus, testing for an effect of single versus redundant stimuli in the TOJ task can indicate whether the RSE arises, at least partly, in this initial common pathway. It should be emphasized, however, that the numerical effect sizes (i.e., in milliseconds) are not necessarily comparable across simple RT and TOJ tasks, so we need not expect the same size redundancy effect on TOJs as on simple RT, even if the redundancy effect is entirely perceptual. Other perceptual manipulations (e.g., intensity) often have effects of different sizes on TOJs and on RTs (e.g., Jaśkowski, 1992; Menendez \& Lit, 1983; Roufs, 1974; Sanford, 1971; but see Roufs, 1963). As will be considered further in the General Discussion section, there are a variety of explanations for such dissociations in effect sizes (e.g., Ejima \& Ohtani, 1989; Miller \& Ulrich, 2003; Neumann, Esselmann, \& Klotz, 1993; Steglich \& Neumann, 2000; Sternberg \& Knoll, 1973; for a review, see Jaśkowski, 1996).

\section{EXPERIMENT 1}

This experiment was conducted as a preliminary examination of the effect of redundant visual signals on TOJs, using a large number of relatively unpracticed participants.

\section{Method}

Apparatus and Stimuli. The participants were tested individually in a dimly illuminated, sound-attenuated experimental chamber. Stimuli were presented and responses and response latencies were recorded by an IBM-PC-compatible microcomputer.

The visual stimuli were white rectangles (luminance approximately $70 \mathrm{~cd} / \mathrm{m}^{2}$ ) presented on the dark background of the computer screen. At the viewing distance of $50 \mathrm{~cm}$, each rectangle was $2.3^{\circ}$ high $\times 2^{\circ}$ wide. In single-stimulus trials, the rectangle appeared $3.4^{\circ}$ to either the left or the right of the central fixation square (edge to edge); in redundant-stimulus trials, rectangles were presented on both sides of fixation. Auditory stimuli were $900-\mathrm{Hz}$ tones presented binaurally over headphones at approximately $50 \mathrm{~dB}$. Both visual and auditory stimuli were presented until the participant made a response.

The participants responded by pressing keys with one or both index fingers on a standard computer keyboard. They responded by pressing the / or the $\mathrm{Z}$ key with the right or the left index finger to indicate whether the visual stimulus or the tone had been presented first, and the assignment of responses to keys was counterbalanced across participants.

Procedure. The participants were 42 first-year psychology students at the University of Otago, who participated in partial fulfillment of an introductory psychology class requirement. Each participant was tested in 12 blocks of trials in a single experimental session lasting approximately $45 \mathrm{~min}$. Each block included 12 trials in which the visual stimulus was the left rectangle, 12 in which it was the right rectangle, and 12 in which both rectangles were presented.

Each trial began with a warning interval marked by the presentation of a fixation square, approximately $0.5^{\circ}$ on a side, in the center of the computer screen. The warning square remained on the screen by itself for a warning interval of $500+E$ msec, where $E$ was a random value sampled in each trial from an exponential distribution with a mean of $500 \mathrm{msec}$. At the end of this warning interval, the visual and auditory stimuli for the trial were presented, either simultaneously or with a small SOA, as will be described below, with the warning square remaining on the screen.

In each trial of the TOJ task, both the auditory stimulus and one of the three possible visual stimuli (i.e., left, right, or redundant) were presented. The SOA, measured from the onset of the auditory stimulus to the onset of the visual stimulus, was varied across trials, using an adaptive staircase procedure with a step size of $16 \mathrm{msec}$ (Kaernbach, 1991). For each type of visual stimulus, two independent staircases were maintained with target percentages of $25 \%$ and $75 \%$ "auditory first" responses. These staircases were set to SOA values of $-100 \mathrm{msec}$ and $+100 \mathrm{msec}$, respectively, at the start of testing for each participant, and the ending SOA value in each staircase for one block was used as the starting SOA value for the next block. The data from the two staircases were combined together to compute the final psychometric function for each participant.

\section{Results}

Figure 1 shows the observed psychometric functions for single and redundant visual stimuli. These observed functions were averaged across observers by Vincentizing (see Ratcliff, 1979). In brief, each observer's observed psychometric function was monotonized using the maximumlikelihood algorithm of Ayer, Brunk, Ewing, Reid, and Silverman (1955). Then linear interpolation was used as necessary to estimate for each observer the SOA corresponding to the $p$ th percentile of the psychometric function, for $p=.05, .15, .25, \ldots, .95$. Finally, the average SOA was computed across observers for each of these percentile values to obtain the Vincentized percentile values.

As is evident from the figure, there was little or no difference between the psychometric functions observed with single versus redundant visual stimuli. Two sets of statistical analyses were computed to compare the TOJs observed in these two conditions. In one set, for each observer, we estimated the value of each psychometric function (i.e., single or redundant) at each of 10 different percentile points from the 5 th to the 95 th percentile in steps of $10 \%$. Paired $t$ tests were then used to compare the single versus the redundant conditions, on average, across participants, separately for each percentile point. Reliable differences were obtained $(p<.05)$ at the 35 th and 45th percentiles; at both of these percentiles, the single-stimulus condition had an SOA approximately $35 \mathrm{msec}$ earlier than that in the redundant condition. These effects are in the direction consistent with a redundancy gain in perceptual speed - that is, the tone can occur slightly earlier in a trial with redundant, rather than single, stimuli and still be judged to have occurred earlier in a fixed proportion of the trials (e.g., 35\%). In addition, $95 \%$ confidence intervals were computed to see how accurately the differences were estimated at each percentile. The confidence intervals indicated that the differences in percentiles were estimated to within approximately $35 \mathrm{msec}$.

In a second set of analyses, each participant's psychometric functions were summarized, for the single and the redundant conditions separately, once using probit analysis and once using the Spearman-Kärber method (see 
Miller \& Ulrich, 2001, for a comparison of these methods). Each method of analysis treats a psychometric function as a cumulative probability distribution (e.g., Finney, 1952; Trevan, 1927) and summarizes it in terms of its central tendency (mean for probit analysis, mean and median for Spearman-Kärber analysis) and variability (standard deviation and difference limen [DL] for both methods). The averages of these summary statistics across participants are shown in Table 1. Paired $t$ tests were computed to check for differences between the single- and the redundant-stimulus conditions, and no significant differences were observed $(p>.15$ in all cases). If redundant stimuli are perceived more rapidly than single stimuli, the means and medians estimated from the redundant-stimulus condition should be larger than those estimated from the single-stimulus condition. The effects are in the direction consistent with faster perception of redundant stimuli for all three measures (probit mean vs. Spearman-Kärber mean vs. Spearman-Kärber median), but never significantly so. There was also a consistent tendency - never significant - for redundant stimuli to yield steeper psychometric functions than did single stimuli, as indexed by the variability measures.

\section{EXPERIMENT 2}

This was essentially a replication of Experiment 1, except that a few participants were tested over 10 sessions to see whether the results would generalize to highly practiced observers.

\section{Method}

The participants were 8 students ( 4 females) recruited on the campus of the University of Otago, and they ranged in age from 19 to 24 years $(M=21.1$ years). Each participated in 10 experimental sessions within a period of 15 days, with at most 2 sessions in a single day, separated by at least $1 \mathrm{~h}$. These participants were paid NZ \$70 for their participation. A 9th participant was also tested, but his data

Table 1

Summary Measures of the Psychometric Functions Obtained With Single and Redundant Visual Stimuli in Experiments 1 and 2

\begin{tabular}{ccccc}
\hline & $\begin{array}{c}\text { Experiment 1 } \\
\text { (Single Session) }\end{array}$ & & $\begin{array}{c}\text { Experiment 2 } \\
\text { (Multisession) }\end{array}$ \\
\cline { 2 - 3 } Single Redundant & & Single Redundant
\end{tabular}

\begin{tabular}{lcccc}
\hline \multicolumn{5}{c}{ Measures of Central Tendency } \\
Probit mean & -64 & -52 & -88 & -84 \\
Spearman-Kärber mean & -63 & -53 & -85 & -86 \\
Spearman-Kärber median & -81 & -58 & -80 & -83 \\
\multicolumn{5}{c}{ Measures of Variability } \\
Probit SD & 228 & 222 & 218 & 202 \\
Probit DL & 154 & 150 & 147 & 136 \\
Spearman-Kärber SD & 171 & 168 & 173 & 157 \\
Spearman-Kärber DL & 146 & 144 & 124 & 121 \\
\hline
\end{tabular}

Note-Summary measures of central tendency are the mean and median, and measures of variability are the standard deviation $(S D)$ and difference limen (DL). The units of all measures are milliseconds. Summary measures were computed using both probit analysis and the Spearman-Kärber method. were excluded because he had great difficulty performing the task accurately (i.e., his psychometric functions were much shallower than those of the other participants). Aside from the repeated testing of individual participants, this experiment was identical to Experiment 1.

\section{Results}

Figure 2 shows the observed psychometric functions for single and redundant visual stimuli, pooling trials across Sessions $3-10 .{ }^{1}$ Clearly, there was little or no difference between the functions for single and redundant stimuli. Although the psychometric functions varied greatly from one observer to the next, there was little difference between the single and the redundant functions for any observer. Paired $t$ tests comparing the single and the redundant conditions showed marginally significant $(p<.10)$ effects of 20 and $14 \mathrm{msec}$ at the 25 th and 75 th percentiles, respectively, with larger SOAs in the redundant conditions at these percentiles, consistent with redundancy gain in detection time. At the 95th percentile, however, a significant effect of $35 \mathrm{msec}$ in the opposite direction was obtained $(p<.05)$. Comparisons of the summary statistics shown in Table 1 showed no hints of differences in measures of central tendency $(p>.1$ for all three measures). There was, however, some evidence of smaller variability with redundant stimuli $(p<.05$ for the Spearman-Kärber estimate of $S D$, and $p<.1$ for the probit estimate of DL).

\section{EXPERIMENT 3}

The small and inconsistent effects of redundant stimuli on TOJs in the first two experiments suggest that, at most, a little of the RSE observed in simple RT arises during the perceptual detection processes common to the TOJ and the RT tasks. In order to strengthen this conclusion, however, it was important to check the size of the RSE on simple RT with the same stimulus conditions as those used in the TOJ tasks of these experiments. That was the purpose of this experiment.

\section{Method}

The participants were 20 individuals from the same pool as that tested in Experiment 1. Each was tested in 12 blocks of trials during a single session lasting approximately $45 \mathrm{~min}$. In 6 blocks, the left and the right rectangles were each presented in eight trials, both were presented in eight trials, and there were four catch trials in which no stimulus was presented. The other 6 blocks included these same trial types plus an additional eight trials in which just an auditory stimulus was presented. This between-blocks comparison was included to see whether there was any effect of requiring participants to monitor for tone onset, because this requirement is necessarily present in the TOJ task but is generally absent from experiments looking at the effects of redundant visual signals on RT. In fact, this manipulation had no effect on the results, so it will not be discussed further. In all blocks, the participants were instructed to respond by pressing the slash key with the right index finger as quickly as possible after the onset of any stimulus.

\section{Results}

The participants responded to all visual and auditory stimuli, yet made false alarms in only approximately $0.4 \%$ 

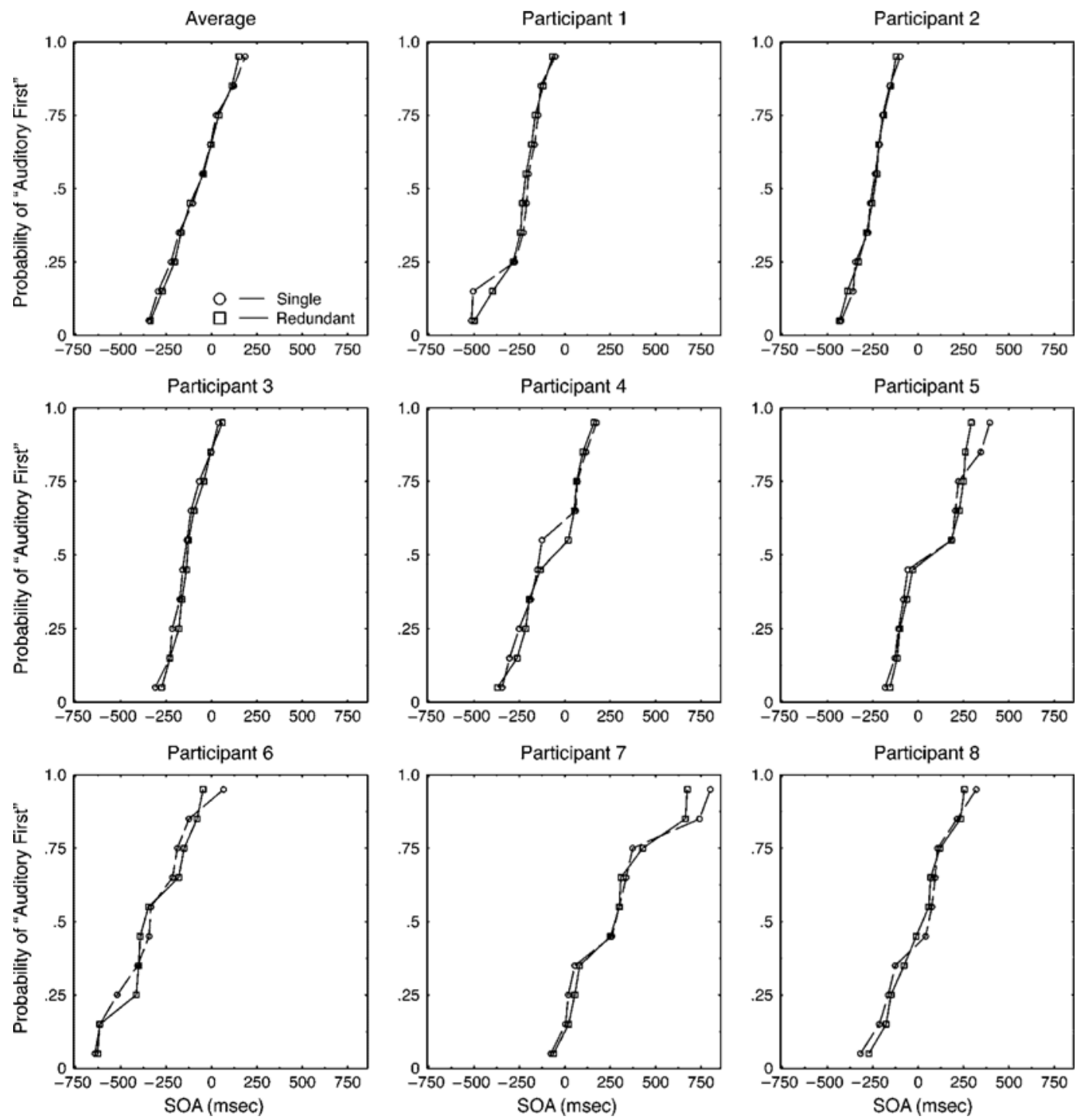

Figure 2. Psychometric functions for single and redundant visual stimuli in Sessions 3-10 of Experiment 2. The upper left panel shows the Vincentized average psychometric functions across all 8 participants, and the other panels show the functions for the 8 individual participants.

of the catch trials. The mean RTs to the left and the right visual stimuli were virtually identical $(M=362$ and $360 \mathrm{msec}$, respectively), and these were significantly longer than RTs to redundant visual stimuli $[M=339 \mathrm{msec}$; $\left.F(2,36)=26.39, M S_{\mathrm{e}}=248, p<.001\right]$. The mean RT to tones was $343 \mathrm{msec}$.

To examine the effects of redundancy at the distributional level, Vincentized RT distributions were computed for the single- and redundant-stimulus conditions, and these are shown in Figure 3. Notably, the 22-msec advantage for redundant stimuli over single stimuli was consistent throughout the full range of the RT distributions, from shortest to longest RTs. In keeping with this shift, the distributions of single- versus redundantstimulus RTs were significantly different $(p<.05)$ by the Kolmogorov-Smirnov test for 10 out of 20 partici- pants and were marginally significant $(p<.10)$ for 1 other. Also shown in the figure is the sum of the Vincentized single-stimulus CDFs, used to test the race model inequality. The inequality was significantly violated at the 5 th percentile $[t(18)=2.875, p<.01]$, indicating that some coactivation was present in these data.

Another measure for indexing the effects of redundancy on RT distributions (e.g., Townsend \& Nozawa, 1995; Wenger \& Townsend, 2000) is the total system capacity up to each time point, $C(t)$, defined as

$$
C(t)=\frac{H_{\mathrm{r}}(t)}{H_{1}(t)+H_{2}(t)} .
$$

$H_{\mathrm{r}}(t)$ is the integrated hazard function for the condition with redundant stimuli- that is, $H_{\mathrm{r}}(t)=\int_{0}^{t} h_{\mathrm{r}}(x) d x-$ 


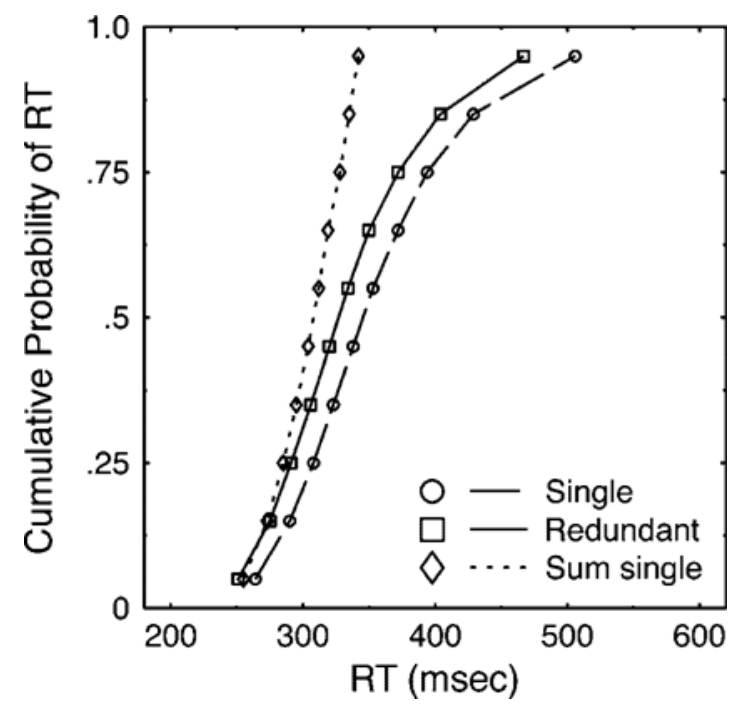

Figure 3. Vincentized cumulative reaction time (RT) distributions for single and redundant visual stimuli in Experiment 3, and the sum of the cumulative probability distributions of the two single-stimulus conditions.

where $h_{\mathrm{r}}(t)$ is the hazard function in this condition (see Luce, 1986; Townsend \& Ashby, 1983). $H_{1}(t)$ and $H_{2}(t)$ are the analogous quantities for the two single-stimulus conditions. With this measure, the value $C(t)=1$ is the maximum value compatible with unlimited-capacity processing models, so observed values of $C(t)>1$ are evidence of supercapacity processing, which is closely related to coactivation (see Townsend \& Nozawa, 1995). Figure 4 shows $C(t)$ functions estimated from the results of this experiment, using the procedure outlined by Wenger and Townsend (2000) with a bin size of 20 msec. Consistent with the results of the CDF analysis, there are strong signs of supercapacity processing in the lower tail of the RT distribution. Over most of the RT distribution, however, $C(t)<1$, which could be a sign that responses to redundant signals are based on less than the sum of the capacities used in responding to single signals. Unfortunately, it is difficult to be sure of this interpretation, because variability in the motor component of the RT also tends to lead to results in which $C(t)<1$. $^{2}$

Comparison of TOJ and RT results. The effect of redundancy on mean RT in this experiment was compared with its effect on mean perceptual latency. ${ }^{3}$ In order to perform such a comparison, the first step was to combine the results from the short- and long-term participants in Experiments 1 and 2 into a single overall measure, $X^{\prime}$, of the effect of redundancy on perceptual latency, which could then be compared with the redundancy effect on RT in Experiment 3. A simple approach to combining these two sets of TOJ results is to average them, obtaining

$$
X^{\prime}=.5 \cdot X_{\mathrm{s}}+.5 \cdot X_{1}
$$

where $X_{\mathrm{s}}$ and $X_{1}$ are the effects of redundancy on TOJs of the short-term and long-term participants in Experi- ments 1 and 2, respectively. The standard error of this quantity can be estimated as

$$
\hat{\sigma}_{X^{\prime}}=\sqrt{.5^{2} \cdot \hat{\sigma}_{X_{\mathrm{s}}}^{2}+.5^{2} \cdot \hat{\sigma}_{X_{1}}^{2}},
$$

where $\hat{\sigma}_{X_{\mathrm{s}}}$ and $\hat{\sigma}_{X_{1}}$ are the observed standard errors of the redundancy effects with the short- and the long-term participants, respectively. The null hypothesis of equal redundancy effects on TOJs and RT can then be tested by computing

$$
\Delta \equiv X^{\prime}-Y,
$$

where $Y$ corresponds to the effect of redundancy on RTs in Experiment 3. The standard error of $\Delta, \hat{\sigma}_{\Delta}$, can be estimated as

$$
\hat{\sigma}_{\Delta}=\sqrt{\hat{\sigma}_{X^{\prime}}^{2}+\hat{\sigma}_{Y}^{2}}
$$

Using this approach yields the estimates $\Delta=-17.7$ and $\hat{\sigma}_{\Delta}=5.788$. The overall $z$ test of the null hypothesis is then given by

$$
z=\frac{\Delta}{\hat{\sigma}_{\Delta}}=-3.058
$$

which has a tail probability considerably less than $1 \%$. Thus, the null hypothesis of equal redundancy effects on mean RT and perceptual latencies can be rejected with the combined data of Experiments 1, 2, and 3.4

\section{EXPERIMENT 4}

Although the results of the first three experiments suggest that redundant stimuli have reliably larger effects on RTs than on TOJs, a subtle artifact in this between-

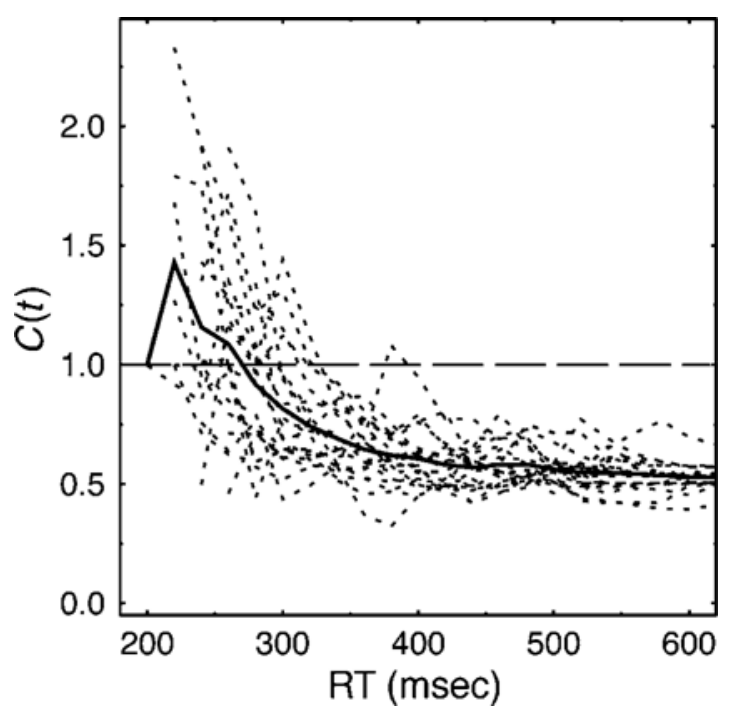

Figure 4. Relative capacity index $C(t)$ as a function of reaction time (RT). The dotted lines display $C(t)$ functions for each individual participant, and the solid line displays the average $C(t)$ across individuals for each value of $t$. The dashed line is a reference line with $C(t)=1$. 
experiments comparison may have been responsible for these results. ${ }^{5}$ The artifact arose because the two types of experiments had different mixtures of visual displays. In the TOJ experiments, trials were equally likely to contain a stimulus on the left, a stimulus on the right, or redundant stimuli. As a result, the a priori likelihood of a given stimulus (e.g., the one on the left) was $2 / 3$, but the likelihood of that stimulus given the other stimulus was only 1/2. In the terms of Mordkoff and Yantis (1991), this reduction in the likelihood of one stimulus given the other represents a negative interstimulus contingency that could interfere with stimulus detection in redundant trials. In the RT experiment, however, there were also some catch trials with no visual stimulus at all, and the presence of these catch trials weakened the negative interstimulus contingency, relative to the TOJ task. It is possible, then, that redundancy gain was larger in the RT task than in the TOJ task because of the change in interstimulus contingencies, rather than because of any inherent differences between the two tasks.

Experiment 4 was carried out to test this possible explanation of the discrepant redundancy effects on RTs versus TOJs. The method was identical to that in Experiment 3, except that no catch trials or auditory stimuli were included. We hoped that random variation in the warning interval-identical to that included in the TOJ experiments - would be sufficient to prevent anticipation responses.

\section{Method}

The participants were 20 right-handed volunteers ( 17 females) between 20 and 30 years of age, recruited at the University of Tübingen. Sixteen participated in partial fulfillment of a curriculum requirement, and the other 4 were paid five Euros each for their participation. Each was tested in 12 blocks of trials during a single session lasting approximately $35-40 \mathrm{~min}$. Each block included 30 trials, equally divided among the three possible stimulus conditions: left, right, or both. The participants were instructed to respond by pressing a designated key with the right index finger as quickly as possible after the onset of any stimulus.

\section{Results}

The participants responded within $100 \mathrm{msec}$ of stimulus onset in approximately $1.8 \%$ of the trials, and these trials were excluded as anticipations. There were no misses. The mean RTs to the left and the right visual stimuli were virtually identical $(M=308$ and $305 \mathrm{msec}$, respectively), and these were significantly longer than RTs to redundant visual stimuli $[M=289 \mathrm{msec} ; F(2,38)=$ 25.67, $\left.M S_{\mathrm{e}}=955, p<.001\right]$. The overall decrease in RT, relative to Experiment 3, may reflect differences in the equipment (e.g., display, keyboard) or in the participant populations. As was the case in Experiment 3, a comparison of Vincentized RT distributions indicated that the effects of redundancy were present throughout the full range of the RT distributions, and the distributions of single- versus redundant-stimulus RTs were significantly different $(p<.05)$ by the Kolmogorov-Smirnov test for 14 out of 20 participants and were marginally significant $(p<.10)$ for 1 other. Unlike the results of Experiment 3, however, the results of this experiment did not produce violations of the race model inequality. Similarly, an analysis of the RT distributions with the $C(t)$ measure, using the same procedure as that in Experiment 3, indicated $C(t)$ functions with the same general shape as those obtained in the earlier experiment, although with noticeably fewer points indicating supercapacity [i.e., $C(t)>1$ ].

As in Experiment 3, the crucial analysis of this experiment compared the observed effect of redundancy gain with that obtained in the TOJ experiments. An analysis using the same method as that employed in the earlier experiment yielded $\Delta=-12.91, \hat{\sigma}_{\Delta}=5.54$, and $z=$ -2.33 . This $z$ value is again highly significant $(p<.01$, one-tailed), so this experiment reinforces the conclusion that redundancy has a larger effect on RTs than on TOJs. In particular, it does not appear that the change in interstimulus contingencies was entirely responsible for the earlier conclusion. Three differences in results in Experiments 3 and 4 do suggest, however, that the change in interstimulus contingencies had an effect in the direction predicted by the model of Mordkoff and Yantis (1991). Specifically, (1) the overall effect of redundancy on mean RT decreased in the latter experiment from 22 to $17 \mathrm{msec}$, although this change was not significant $[F(1,38)=$ $\left.2.65, M S_{\mathrm{e}}=86.81, .15>p>.10\right]$; (2) violations of the race model inequality were present in Experiment 3, but not in Experiment 4; and (3) there was stronger evidence of supercapacity processing of redundant stimuli in Experiment 3 than in Experiment 4.

\section{GENERAL DISCUSSION}

The large effects of redundancy on simple RTs in Experiments 3 and 4 contrast sharply with its small and inconsistent effects on TOJs in Experiments 1 and 2. Although participants can clearly respond more rapidly to redundant visual stimuli than to single visual stimuli, there is very little evidence that the redundant stimuli are perceived more quickly at the level of the perceptual latencies determining TOJs. Therefore, these results argue against a relatively early perceptual source of redundancy gain in simple RT and, instead, favor a relatively late account in which redundancy affects decision-making or motor processes. This conclusion is strengthened by evidence that perceptual manipulations (e.g., stimulus intensity) have strong effects on TOJs (e.g., Sanford, 1971). To the extent that perceptual manipulations generally affect TOJs, the fact that a manipulation does not affect TOJs suggests that it does not have a perceptual effect.

As was mentioned in the introduction, a variety of perceptual manipulations (e.g., intensity) have larger effects on RT than on TOJs, and it is worthwhile to consider whether current theoretical accounts of these dissociations (see Jaśkowski, 1996, 1999, for reviews) could be extended to account for the virtual lack of redundancy effect on TOJs. One account is that the extra effects in RT tasks reflect postperceptual processes, but of course this account is completely consistent with our conclu- 
sion that the redundancy effect is postperceptual. A second account explains the dissociations in terms of an influence of stimulus offsets on TOJs, but not on RTs; this account, however, does not apply to the present responseterminated stimuli. A third account is that observers in TOJ experiments compensate somewhat for perceptual manipulations by paying more attention to the perceptually weaker stimulus (e.g., lower intensity). Such attention shifting could not account for the absence of a redundancy effect in the present TOJ experiments, however, because there would be no way to pay extra attention to single stimuli, as opposed to redundant ones. Moreover, recent tests of this account do not support the idea that the distribution of attention is influenced by stimulus intensity in TOJ tasks (Jaśkowski \& Verleger, 2000).

Three more accounts of the dissociation appear able to explain a finding of smaller redundancy effects in TOJs than in RTs, although none of these seems able to explain the absence of such effects. One such account, referred to by Jaśkowski (1996, p. 716) as the "hypothesis of different time-markers," is based on the idea that observers rely on different aspects of perceptual processing in RT tasks than in TOJ tasks. For example, Sternberg and Knoll (1973) suggested that RT responses may depend on the time at which perceptual activation crosses a threshold, whereas TOJs may depend on the time at which such activation reaches its peak. ${ }^{6}$ This account predicts that perceptual manipulations would affect TOJs less than RTs, but it does not predict that effects on TOJs would disappear completely unless the latency of peak activation is insensitive to the perceptual manipulation, which seems unlikely. A second such account, described by Jaśkowski (1999) as the "channel coactivation hypothesis," is based on the idea that intersensory interactions between two stimuli speed the detection of both in a TOJ task (see Bernstein, 1970). Again, these interactions would eliminate some of the effect of an experimental manipulation but would not make it disappear entirely. Finally, Miller and Ulrich (2003) developed an account based on the different variabilities of times needed to transmit perceptual signals to a conscious decision system and to a system responsible for generating motor responses. Their account also predicts that the effects of perceptual manipulations would be smaller in TOJ tasks than in RT tasks but would not disappear completely.

Because two visual stimuli were presented in redundant trials, there is a special variant of the hypothesis of different time markers that could account for the lack of redundancy effect on TOJs in these experiments. Suppose that in redundant trials, observers use some form of an average detection time of the two visual stimuli as the estimated visual latency for the TOJ, whereas they use the first-detected stimulus to generate a response in the RT task. In the TOJ task, the average of the two visual latencies in redundant trials would be the same as the average visual latency in single trials, so redundant displays would not appear to have earlier onsets than do single displays, on average. The idea that visual latency might be determined by the average, rather than the min- imum, detection time for two visual stimuli receives some indirect support from evidence that latency judgments are influenced by the rise times and durations of stimuli, and not only by their onset times (e.g., Jaśkowski, 1993), because this evidence indicates that sensory events following initial onset can influence perceived onset time.

Although this variant of the hypothesis of different time markers accounts for the equal means of the psychometric functions obtained with single and redundant visual stimuli, it seems rather unlikely in view of the similar variances of these functions. Assuming that the two visual latencies in redundant trials have a correlation less than 1 , averaging them would produce a lower variance estimate of the visual onset time than is provided by a single-stimulus display. This, in turn, would reduce the variability of the psychometric function in redundant trials relative to single trials. Table 1 , however, provides little evidence of the dramatic reduction in variability predicted by this account.

Despite the lack of a clear redundancy effect in the TOJ task, it is impossible to conclude that redundancy has no effect on any perceptual process, because it is possible that the simple RT task involves perceptual processes other than or beyond those required for the TOJ task. In that case, redundancy could still affect some perceptual process that was used in the simple RT task, but not in the TOJ task. For example, Neumann and his colleagues (e.g., Neumann, 1990; Steglich \& Neumann, 2000; Tappe et al., 1994) have suggested that, after an initial common perceptual processing pathway, incoming stimuli may be processed through two separate perceptual processing streams to influence motor responses and conscious awareness. According to Steglich and Neumann (2000), these two processing streams might correspond to separate dorsal and ventral perceptual pathways (see Goodale \& Humphrey, 1998; Milner \& Goodale, 1993). Within the context of this two-pathway model, the present results would imply that redundancy influenced neither the initial common pathway nor the pathway leading to conscious experience, although it could still influence perceptual processes driving the motor control system via what Neumann calls direct parameter specification. Even on this view, however, redundancy would be a fairly unusual perceptual manipulation, in that it would affect only the direct parameter specification pathway but not the initial common pathway or the pathway leading to conscious experience, whereas other perceptual manipulations (e.g., intensity) generally do have some influence on the common pathway and, hence, on TOJs. ${ }^{7}$

Although the present work provides evidence that the RSE does not arise during the perceptual processing required in the TOJ task, the locus of this effect remains unclear. Given previous evidence that the effect is not motor (e.g., Miller et al., 2001; Mordkoff et al., 1996), the suggestion from all of the findings together is that the RSE must arise in some fairly central process occurring after the common perceptual pathway used in both 
simple RT tasks and TOJ tasks but before the stage of motor activation. Some clues as to the nature of this process may be provided by the psychophysiological effects of redundancy, noted earlier (Miniussi et al., 1998; Murray et al., 2001). Thus, even though the present experiments provide new information that helps to localize the RSE after early perceptual processes, further research will be needed to isolate the exact processes responsible for this effect.

\section{REFERENCES}

Ashby, F. G., \& TownsEnd, J. T. (1986). Varieties of perceptual independence. Psychological Review, 93, 154-179.

Ayer, M., BrunK, H. D., Ewing, G. M., Reid, W. T., \& Silverman, E. (1955). An empirical distribution function for sampling with incomplete information. Annals of Mathematical Statistics, 26, 641-647.

BARR, M. (1983). A comparison of reaction-time and temporal-order judgment estimates of latency to sinusoidal gratings [Abstract]. Perception, 12, A7.

Bernstein, I. H. (1970). Can we see and hear at the same time? Some recent studies of intersensory facilitation of reaction time. Acta Psychologica, 33, 21-35.

Blake, R., MarTens, W., Garrett, A., \& Westendorf, D. (1980). Estimating probability summation for binocular reaction time data. Perception \& Psychophysics, 27, 375-378.

Cavina-Pratesi, C., Bricolo, E., Prior, M., \& Marzi, C. A. (2001). Redundancy gain in the stop-signal paradigm: Implications for the locus of coactivation in simple reaction time. Journal of Experimental Psychology: Human Perception \& Performance, 27, 932-941.

Colonius, H. (1990). Possibly dependent probability summation of reaction time. Journal of Mathematical Psychology, 34, 253-275.

Craig, J. C., \& Busey, T. A. (2003). The effect of motion on tactile and visual temporal order judgments. Perception \& Psychophysics, $\underline{\mathbf{6 5}}$ 81-94.

DIEDERICH, A. (1992). Intersensory facilitation: Race, superposition, and diffusion models for reaction time to multiple stimuli. Frankfurt: Peter Lang.

DiEDERICH, A., \& Colonius, H. (1987). Intersensory facilitation in the motor component? A reaction time analysis. Psychological Research, 49, 23-29.

DONDERS, F. C. (1969). On the speed of mental processes [Over de snelheid van psychische processen]. (W. G. Koster, Trans.). In W. G. Koster (Ed.), Attention and performance II (pp. 412-431). Amsterdam: North-Holland. [Original work published 1868]

EgETH, H. E., \& MoRdKOFF, J. T. (1991). Redundancy gain revisited: Evidence for parallel processing of separable dimensions. In J. R. Pomerantz \& G. R. Lockhead (Eds.), The perception of structure (pp. 131-140). Washington, DC: American Psychological Association.

EJIMA, Y., \& OHTANI, Y. (1989). Analysis of simple reaction time to a sinusoidal grating by means of a linear filter model of the detection process. Perception \& Psychophysics, 46, 119-126.

FINNEY, D. J. (1952). Probit analysis: A statistical treatment of the sigmoid response curve (2nd ed.). Cambridge: Cambridge University Press.

Finney, D. J. (1978). Statistical method in biological assay. London: Griffin.

Forster, B., Cavina-Pratesi, C., Aglioti, S. M., \& Berlucchi, G. (2002). Redundant target effect and intersensory facilitation from visual-tactile interactions in simple reaction time. Experimental Brain Research, 143, 480-487.

GIBSON, B. S., \& EGETH, H. (1994). Inhibition and disinhibition of return: Evidence from temporal order judgments. Perception \& Psychophysics, 56, 669-680.

GIRAY, M., \& UlRich, R. (1993). Motor coactivation revealed by response force in divided and focused attention. Journal of Experimental Psychology: Human Perception \& Performance, 19, 12781291.

Goodale, M. A., \& Humphrey, G. K. (1998). The objects of action and perception. Cognition, 67, 181-207.
Grice, G. R., Canham, L., \& Boroughs, J. M. (1984). Combination rule for redundant information in reaction time tasks with divided attention. Perception \& Psychophysics, 35, 451-463.

Hershenson, M. (1962). Reaction time as a measure of intersensory facilitation. Journal of Experimental Psychology, 63, 289-293.

JAŚKOWSKI, P. (1992). Temporal-order judgment and reaction time for short and long stimuli. Psychological Research, 54, 141-145.

JAŚKowsKI, P. (1993). Temporal-order judgment and reaction time to stimuli of different rise times. Perception, 22, 963-970.

JAŚKOWSKI, P. (1996). Simple reaction time and perception of temporal order: Dissociations and hypotheses. Perceptual \& Motor Skills, $\mathbf{8 2}_{2}$ 707-730.

JAŠKOWSKI, P. (1999). Reaction time and temporal-order judgment as measures of perceptual latency: The problem of dissociations. In G. Aschersleben, T. Bachmann, \& J. Müsseler (Eds.), Cognitive contributions to the perception of spatial and temporal events (pp. 265282). Amsterdam: Elsevier.

JAŚKOWSKI, P., \& VERLEGER, R. (2000). Attentional bias toward lowintensity stimuli: An explanation for the intensity dissociation between reaction time and temporal order judgment? Consciousness \& Cognition, 9, 435-456.

KAERNBACH, C. (1991). Simple adaptive testing with the weighted up-down method. Perception \& Psychophysics, 49, 227-229.

LogAN, G. D. (1994). On the ability to inhibit thought and action: A users' guide to the stop signal paradigm. In D. Dagenbach \& T. H. Carr (Eds.), Inhibitory processes in attention, memory, and language (pp. 189-239). New York: Academic Press.

LoRD, F. M., \& Novick, M. R. (1968). Statistical theories of mental test scores. Reading, MA: Addison-Wesley.

LuCE, R. D. (1986). Response times: Their role in inferring elementary mental organization. Oxford: Oxford University Press.

May, J. G., Martin, F., MacCana, F., \& Lovegrove, W. J. (1988). The effects of spatial frequency and temporal waveform on three measures of temporal processing. Journal of General Psychology, 115, 293-306.

Menendez, A., \& Lit, A. (1983). Effects of test flash and steady background luminance on simple visual reaction time and perceived simultaneity [Abstract]. Investigative Ophthalmology \& Visual Science, 24, 95 .

Meyer, D. E., Osman, A. M., Irwin, D. E., \& Yantis, S. (1988). Modern mental chronometry. Biological Psychology, 26, 3-67.

Miller, J. [O.] (1982). Divided attention: Evidence for coactivation with redundant signals. Cognitive Psychology, 14, 247-279.

Miller, J. [O.], \& UlRich, R. (2001). On the analysis of psychometric functions: The Spearman-Kärber method. Perception \& Psychophysics, 63, 1399-1420.

MILLER, J. [O.], \& ULRICH, R. (2003). Simple reaction time and statistical facilitation: A parallel grains model. Cognitive Psychology, $\mathbf{4 6}$, 101-151.

MILLER, J. [O.], \& ULRICH, R. (2004). A computer program for SpearmanKärber and probit analysis of psychometric function data. Behavior Research Methods, Instruments, \& Computers, 36, 11-16.

Miller, J. [O.], Ulrich, R., \& Lamarre, Y. (2001). Locus of the redundant-signals effect in bimodal divided attention: A neurophysiological analysis. Perception \& Psychophysics, 63, 555-562.

Milner, D. A., \& Goodale, M. A. (1993). Visual pathways to perception and action. In T. P. Hicks, S. Molotchnikoff, \& T. Ono (Eds.), Progress in brain research (Vol. 95, pp. 317-337). Amsterdam: Elsevier.

Miniussi, C., Girelli, M., \& Marzi, C. A. (1998). Neural site of the redundant target effect: Electrophysiological evidence. Journal of Cognitive Neuroscience, 10, 216-230.

MoRdKOFF, J. T., \& Miller, J. [O.] (1993). Redundancy gains and coactivation with two different targets: The problem of target preferences and the effects of display frequency. Perception \& Psychophysics, 53, 527-535.

Mordkoff, J. T., Miller, J. O., \& Roch, A. C. (1996). Absence of coactivation in the motor component: Evidence from psychophysiological measures of target detection. Journal of Experimental Psychology: Human Perception \& Performance, 22, 25-41.

MORDKOFF, J. T., \& YANTIS, S. (1991). An interactive race model of divided attention. Journal of Experimental Psychology: Human Perception \& Performance, 17, 520-538. 
MordKoff, J. T., \& YAntis, S. (1993). Dividing attention between color and shape: Evidence of coactivation. Perception \& Psychophysics, 53, 357-366.

MURRAY, M. M., FoXe, J. J., Higgins, B. A., JAVITT, D. C., \& Schroeder, C. E. (2001). Visuo-spatial neural response interactions in early cortical processing during a simple reaction time task: A high-density electrical mapping study. Neuropsychologia, 39, 828-844.

NeumanN, O. (1990). Direct parameter specification and the concept of perception. Psychological Research, 52, 207-215.

Neumann, O., Esselmann, U., \& Klotz,W. (1993). Differential effects of visual-spatial attention on response latency and temporalorder judgment. Psychological Research, 56, 26-34.

PARKer, D. M., \& Dutch, S. (1987). Perceptual latency and spatial frequency. Vision Research, 27, 1279-1283.

Plat, F. M., PraAmstra, P., \& HorstinK, M. W. I. M. (2000). Redundantsignals effects on reaction time, response force, and movement-related potentials in Parkinson's disease. Experimental Brain Research, 130 , 533-539.

RAAB, D. H. (1962). Statistical facilitation of simple reaction times. Transactions of the New York Academy of Sciences, 24, 574-590.

RATCLIFF, R. (1979). Group reaction time distributions and an analysis of distribution statistics. Psychological Bulletin, 86, 446-461.

Roufs, J. A. J. (1963). Perception lag as a function of stimulus luminance. Vision Research, 3, 81-91.

Roufs, J. A. J. (1974). Dynamic properties of vision: V. Perception lag and reaction time in relation to flicker and flash thresholds. Vision Research, 14, 853-869.

SANFORD, A. J. (1971). Effects of changes in the intensity of white noise on simultaneity judgements and simple reaction time. Quarterly Journal of Experimental Psychology, 23, 296-303.

Schwarz, W. (1989). A new model to explain the redundant-signals effect. Perception \& Psychophysics, 46, 498-500.

Schwarz, W. (1994). Diffusion, superposition, and the redundant-targets effect. Journal of Mathematical Psychology, 38, 504-520.

Schwarz, W., \& IschebeCK, A. (1994). Coactivation and statistical facilitation in the detection of lines. Perception, 23, 157-168.

Shore, D. I., Spence, C., \& Klein, R. M. (2001). Visual prior entry. Psychological Science, 12, 205-212.

SpENCE, C., Shore, D. I., \& KLEIN, R. M. (2001). Multisensory prior entry. Journal of Experimental Psychology: General, 130, 799-832.

Steglich, C., \& NeumanN, O. (2000). Temporal, but not spatial, context modulates a masked prime's effect on temporal order judgment, but not on response latency. Psychological Research, 63, 36-47.

Stelmach, L. B., \& Herdman, C. M. (1991). Directed attention and perception of temporal order. Journal of Experimental Psychology: Human Perception \& Performance, 17, 539-550.

StERNBERG, S., \& KNOLL, R. L. (1973). The perception of temporal order: Fundamental issues and a general model. In S. Kornblum (Ed.), Attention and performance IV (pp. 629-685). New York: Academic Press.

TAPPE, T., NiePel, M., \& Neumann, O. (1994). A dissociation between reaction time to sinusoidal gratings and temporal-order judgment. Perception, 23, 335-347.

ToDD, J. (1912). Reaction to multiple stimuli. Archives of Psychology, 3, 1-65.

Townsend, J. T., \& AshBy, F. G. (1983). The stochastic modeling of elementary psychological processes. Cambridge: Cambridge University Press.

Townsend, J. T., \& NozAWA, G. (1995). Spatio-temporal properties of elementary perception: An investigation of parallel, serial, and coactive theories. Journal of Mathematical Psychology, 39, 321-359.

Townsend, J. T., \& NozAWA, G. (1997). Serial exhaustive models can violate the race model inequality: Implications for architecture and capacity. Psychological Review, 104, 595-602.

Trevan, J. W. (1927). The error of determination of toxicity. Proceedings of the Royal Society of London: Series B, 101, 483-514.

Tukey, J. W. (1948). Approximate weights. Annals of Mathematical Statistics, 19, 91-92.

ULRICH, R., \& GIRAY, M. (1986). Separate-activation models with vari- able base times: Testability and checking of cross-channel dependency. Perception \& Psychophysics, 39, 248-254.

Wenger, M. J., \& TownSEND, J. T. (2000). Basic response time tools for studying general processing capacity in attention, perception, and cognition. Journal of General Psychology, 127, 67-99.

\section{NOTES}

1. The first two sessions were excluded, so that only trials from reasonably well-practiced participants would be included. In fact, this had little impact on the results, because virtually identical psychometric functions were computed in a separate analysis including all the sessions.

2. To elaborate, most RT theorists (e.g., Luce, 1986) postulate that observable RT is the sum $T+M$, where $T$ is the time needed for the processing of interest in the task and $M$ is a subsequent motor time needed for response execution. In the redundancy gain paradigm, even if $C(t)=1$ holds at the level of the random variable $T$ that is of main interest, one can obtain $C(t)<1$ at the level of the observable values of $T+M$ if $M$ has some variance and is imperfectly correlated with $T$. A small numerical example suffices to demonstrate this possibility. Suppose that the detection times for two single stimuli are exponential random variables $T_{1}$ and $T_{2}$, both with a rate of $1 / 100$, and that the detection time for redundant stimuli is exponential with a rate of $2 / 100$, in keeping with an unlimited-capacity parallel model. These random variables yield $C(t)=1$ for all $t$ s. Now suppose that the observable RT in each stimulus condition is the sum of the appropriate detection time plus an independent motor time, $M$, which we assume for computational convenience to be exponentially distributed with a rate of $1 / 50$. These observable RTs would yield $C(t)<1$ for all $t \mathrm{~s}$, and $C(t)<0.90$ for most values of $t$. Thus, variability of the motor time contaminates $C(t)$, just as it can obscure violations of the race model inequality (Miller \& Ulrich, 2003; Ulrich \& Giray, 1986).

3. The analysis reported here used mean perceptual latencies estimated with the Spearman-Kärber method (see Miller \& Ulrich, 2001, 2004), because these estimates are more directly comparable to mean RTs than are alternative estimates of perceptual latencies (Sternberg \& Knoll, 1973). Similar results were obtained using mean perceptual latencies estimated via probit analysis, however (e.g., Finney, 1978).

4. Two other, more complicated approaches to testing this null hypothesis suggest giving different weights to the results of Experiments 1 and 2. With these approaches, the values of .5 in Equations 3 and 4 would be replaced with some values of $w$ for the short-term results and $1-w$ for the long-term results. One approach, based on test theory (Lord \& Novick, 1968), suggests that the optimal weighting would be given by $w=42 /(80+42)$, because the short- and long-term averages were based on 42 and 80 sessions of data, respectively. The other approach attempts to estimate the optimal value of $w$ from the observed standard errors $\hat{\sigma}_{X_{5}}$ and $\hat{\sigma}_{X_{1}}$. Tukey (1948) showed that the optimal value is $w=\sigma_{X_{1}}^{2} /\left(\sigma_{X_{1}}^{2}+\sigma_{X_{\mathrm{s}}}^{2}\right)$, where $\sigma_{X_{\mathrm{s}}}$ and $\sigma_{X_{1}}$ are the true standard deviations of $X_{\mathrm{s}}$ and $X_{1}$, and this quantity can be estimated using the observed standard errors of $\hat{\sigma}_{X_{s}}$ and $\hat{\sigma}_{X_{1}}$. Fortunately, both of these alternative approaches also allow rejection of the null hypothesis of equal redundancy effects on RT and perceptual latencies, just like the simple averaging approach described in the text.

5 . We are indebted to J. Toby Mordkoff for pointing out this potential artifact.

6. In an elaboration of this idea, Stelmach and Herdman (1991) suggested that TOJs could depend on a comparison of the full temporal profiles of the activations produced by two stimuli, rather than just the peaks. They did not explicitly consider how RT might be determined, however.

7. There have been some reports that RTs, but not TOJs, are affected by the spatial frequency composition of visual stimuli (e.g., Barr, 1983; May, Martin, MacCana, \& Lovegrove, 1988). In fact, however, it appears that spatial frequency does affect TOJs when stimulus edges are controlled appropriately (Tappe et al., 1994).

(Manuscript received December 30, 2002; revision accepted for publication September 12, 2003.) 